This is the final peer-reviewed accepted manuscript of:

F. Tonini, M. Fiorani, M. Furdek, C. Raffaelli, L. Wosinska and P. Monti, "Radio and Transport Planning of Centralized Radio Architectures in 5G Indoor Scenarios," in IEEE Journal on Selected Areas in Communications, vol. 35, no. 8, pp. 1837-1848, Aug. 2017

The final published version is available online at:

https://doi.org/10.1109/JSAC.2017.2710582

Rights / License:

The terms and conditions for the reuse of this version of the manuscript are specified in the publishing policy. For all terms of use and more information see the publisher's website. 


\title{
Radio and Transport Planning of Centralized Radio Architectures in 5G Indoor Scenarios
}

\author{
Federico Tonini, Matteo Fiorani, Marija Furdek, Carla Raffaelli, Lena Wosinska, Paolo Monti
}

\begin{abstract}
Providing high capacity to the end users is one of the main challenges for the fifth generation (5G) of mobile networks. The users' habit to consume online contents indoor makes the outdoor-to-indoor capacity provisioning impractical, especially when the high frequency bands proposed for $\mathbf{5 G}$ are employed. The Centralized Radio Architecture (CRA) is an inbuilding solution which relies on the centralization of baseband processing functions, fully or partly allowing for centralized cell management while providing signals directly inside the buildings. On the other hand, the massive deployment of CRAs in urban areas may yield to unacceptably high installation costs, due to the radio network equipment to be activated.

To make CRAs appealing to mobile operators, we propose different deployment strategies to minimize the CRA deployment cost. We define the remote radio unit placement (RRUP) problem and formulate it as an Integer Linear Program (ILP), obtaining optimal deployment solutions in small urban residential scenarios. We prove the RRUP problem to be NP-hard, requiring heuristic approaches to solve large problem instances. To this end, we propose an effective and scalable heuristic for minimizing the amount of radio equipment required to deploy CRAs in large urban areas.
\end{abstract}

Index Terms-5G, Fronthaul, Backhaul, Centralized radio architecture (CRA), Indoor network deployment, Cost optimization.

\section{INTRODUCTION}

$\mathbf{T}$ HE fifth generation $(5 \mathrm{G})$ of mobile networks is expected to cater for an immense traffic growth with unprecedented bandwidth requirements, likely to exceed 60 exabytes/month in 2022 [1]. Many solutions have been proposed to cope with this growth. High number of antennas, beamforming techniques, base station densification, and millimeter waves (mmWaves) can drastically improve the data rate delivered to users thanks to an enhanced spectral/spatial efficiency, and a larger bandwidth. Centralization and virtualization of baseband processing functions, separation of control and data planes, and self-backhauling have been identified as enabling technologies for $5 \mathrm{G}$ communication [2].

In $5 \mathrm{G}$ scenarios with an extremely high number of base stations, where the interference may be a limiting factor, baseband centralization could be exploited to increase the performance of the network and meet the $5 \mathrm{G}$ requirements at a lower cost, which is a target of great interest for mobile

F. Tonini, and C. Raffaelli are with the DEI, University of Bologna, Bologna, Italy (e-mail: \{f.tonini, carla.raffaelli\}@unibo.it)

M. Furdek, P. Monti, L. Wosinska are with the Department of Communication Systems (CoS), KTH Royal Institute of Technology, Kista, Sweden (e-mail: \{marifur, wosinska, pmonti\}@kth.se)

M. Fiorani, was with the Department of Communication Systems (CoS), KTH Royal Institute of Technology, he is currently with Ericsson Research, Stockholm, Sweden (e-mail: matteo.fiorani@ericsson.se) network operators. In baseband centralization, the functional blocks of traditional base stations (BSs) are divided in two parts: (i) the Remote Radio Units (RRUs), placed close to the antennas that perform analog signal processing, and (ii) the BaseBand Units (BBUs), that perform digital baseband processing (e.g., interference management and cell coordination) and that are aggregated in selected locations (i.e., BBU Hotels). BBU Hotels can share the same power supply and cooling infrastructure, with additional benefits in terms of cost and energy consumption [3], [4]. An evolution of BBU hotelling concept is the so-called Centralized/Cloud Radio Access Network (C-RAN). In particular, in the centralized approach baseband functions are performed by very efficient proprietary hardware, while the Cloud philosophy proposes to virtualize those functions in general purpose hardware. Covering a wide area with many BBUs (e.g., in the order of thousands), either virtualized or physically located in the same place, has many advantages (e.g., in terms of cost and efficient resource usage) but may add computational complexity due to data exchange and processing for interference management techniques. Proof of concept for centralized-RAN with limited baseband centralization can be found in literature [5], while for hotels employing a large number of BBUs this aspect needs further investigation. In a C-RAN, the link between RRUs and BBUs (i.e., referred to as fronthaul) requires high capacity. The most popular fronthaul data transmission choice among vendors is digital radio-over-fiber using a standard radio interface, i.e., common public radio interface (CPRI). Recently, different functional splits have been proposed aiming at reducing the amount of resources required by fronthaul links. These functional splits break the RRU-BBU paradigm, moving some of the baseband functions into the RRU. Depending on the split, different requirements must be met in terms of latency and bit-rate, relaxing the traditional fronthaul requirements [6]. In this view, standardization bodies are working on the definition of a new interface called eCPRI [7].

The users' habit to consume online contents indoor requires tailored solutions to meet the $5 \mathrm{G}$ requirements in indoor areas in a cost efficient way. In such circumstances, outdoor-toindoor capacity provisioning may be impractical, especially with the high frequency bands proposed for 5G [8]. Selfbackhauling, which reuse the radio spectral resources to send backhaul data in-band, seems a promising solution [9]. In conjunction with indoor small cells, centralized architectures like ultra high density distributed antenna system (DAS) can be deployed to provide larger data rates [10], [11]. However, due to the large amount of network equipment, a massive deployment of such networks may be extremely expensive, 
if not planned carefully. To reduce network cost, the reuse of existing in-building infrastructure (e.g., already deployed LAN cables) can be combined with efficient deployment strategies to drastically reduce the amount of necessary network equipment.

Motivated by these observations, we recently proposed an optimization approach for minimizing the amount of radio equipment in a centralized architecture to provide indoor coverage in small residential areas [12]. This architecture, called centralized radio architecture (CRA), embodies the benefits of centralized baseband thanks to the separation of RRUs and BBUs. In addition, a CRA decouples antennas from RRUs and allows for RRU placement either in indoor or outdoor cabinets where they can be shared by antennas placed in different buildings. In a CRA, RRUs communicates with antennas through intermediate frequency signals over copper cables, allowing the reuse of existing in-building copper cables. In our preliminary study, the RRU placement (RRUP) problem was formulated as an Integer Linear Program (ILP) and the initial results indicated a significant reduction in the required amount of network components and in the overall network cost compared to existing deployment solutions.

Since an ILP is able to solve only limited size problem, this paper extends the study in [12] to solve larger size instances. This is done by proposing an efficient heuristic algorithm for cost-minimizing CRA deployment in urban scenarios. The RRUP problem can be divided into two parts: (i) finding the minimum number of RRU cabinets to be activated, and (ii) placing the minimum number of RRUs at each cabinet. The first sub-problem (i.e., selecting a minimum set of RRU cabinets) is equivalent to the Set Cover Problem, a wellknown NP-hard optimization problem. The proposed heuristic strategy shows how CRA networks can be deployed in a more efficient way than conventional deployment strategies. Results show remarkable cost savings that may motivate operators to consider centralized networks as a concrete solution for $5 \mathrm{G}$ indoor scenarios.

The rest of the paper is organized as follows. Section II reviews the state of the art in CRA deployments. Section III provides a detailed description of the CRA technology and a formal definition of the RRUP problem, whose proof of NPhardness is presented in the Appendix. Section IV presents the framework comprising the ILP model and a new heuristic approach applicable to urban scenarios. Section V evaluates the performance of both the ILP and the heuristic in district and urban residential scenarios while Section VI concludes the paper.

\section{RELATED WORK}

The origins of the CRA concept can be traced back to [13], where the authors proposed a new paradigm called FemtoWoC (Femto Wireless over Cable) that leaves the analog $\mathrm{RF}$ processing (i.e., antenna and analog-to-analog converter) at the in-home device, while the PHY/MAC functionalities are moved to a remote location (e.g., the operator Central Office). Although the architecture did not introduce a real separation of radio and baseband units, it is noteworthy as the authors also carried out a detailed throughput analysis of the link between antennas and remote locations, which resembles the antennaRRU link in a CRA.

A CRA-based system was introduced in [14], where the authors proposed to use Ethernet cables to carry intermediate frequency (IF) signals between antennas and RRUs. The experimental results presented in the article show that IF signals can be sent over Cat. 6 a cables over distances longer than 100 m. LTE signal propagation over Ethernet cables (Cat-5/6/7) is analyzed in [15], where the authors analyzed the maximum available bandwidth and the maximum number of antenna flows that can be transported over LAN cables of different lengths (up to $200 \mathrm{~m}$ ). Results showed that for $75 \mathrm{~m}$ almost no degradation is experienced in Ethernet cables, and at least 60 antennas using $20 \mathrm{MHz}$ LTE channels can be served by a single $100 \mathrm{~m}$ Ethernet cable, with this number decreasing rapidly for larger distances.

The works in [16], [17] propose a mobile indoor system architecture based on the CRA concept, where antennas are placed indoor and connected to the RRUs located in digital subscriber line access multiplexer (DSLAM) cabinets through twisted pair cables of the public switched telephone network (PSTN) copper plant. The authors analyzed the coexistence of DSL and mobile signals over the same cables and determined the maximum distance over which the radio signals can be transmitted and received, considering the 3GPP requirements. In [18], [19] the authors proposed crosstalk mitigation and cancellation techniques for LTE over copper cables applicable to the CRA architecture. Results show that it is possible to transmit analog radio signals over twisted pairs copper lines in ranges of $300 \mathrm{~m}$.

All the works mentioned so far studied the feasibility and performance of CRAs, focusing mainly on the physical layer aspects of their architecture and on their ability to coexist with already deployed DSL systems. No considerations were made on the challenges related to CRA deployment. Some aspects of CRAs are similar to C-RAN, e.g., they both leverage on the baseband centralization concept and make use of fronthaul links. Optimization strategies for Centralized-RAN deployment, such as the ones focusing on the BBU placement problem, can be found in [20], [21]. In [20], the authors proposed an ILP-based energy efficient BBU placement and showed that energy savings can be achieved by using BBU hoteling architectures. In [21], the authors proposed a BBU placement approach for mobile aggregation networks aimed at minimizing either the number of fibers or the number of BBU Hotels. The study outlined that a higher degree of BBU centralization can be achieved at the expense of an increased total amount of optical fibers needed to carry the fronthaul traffic.

The works above focus only on the minimization of the number of BBU hotels. On the other hand, centralization of the BBU functionalities affects the amount of fibers deployed (i.e., fronthaul traffic is carried over longer distances) and consequently the network deployment cost. The centralization of baseband functions also poses strict requirements on fronthaul link capacity, especially when many antenna flows have to be carried by a single fiber, which may become the limiting factor 
of this architectural solution. For this reason, many works on different functional splits have been proposed recently. A comprehensive overview of optical transport solutions to carry fronthaul data under different baseband splits is provided in [22], while [6] specifies the requirements of the different splits on fronthaul and transport links and provides guidelines for virtualization of the network functions in different scenarios. In [23] the authors study the requirements for a converged fronthaul and backhaul network architecture in a cloud radio access network implementing a flexible functional split. The authors perform a comparison of the different splits to analyze their impact in terms of required throughput and latency as well as protocol and infrastructure requirements. In order to reduce the cost of the fronthaul network, CPRI flows can be encapsulated in Ethernet frames, allowing for exploitation of the existing commercial Ethernet interfaces and switching equipment to carry fronthaul traffic. A performance evaluation of CPRI over Ethernet frames in terms of delay and jitter is presented in [24].

While in the case of Centralized-RAN the position of the BBUs is the only variable to consider, the position of the RRUs is an additional key parameter to consider in CRA, which increases the complexity of the network deployment problem. So far, only a few works on CRA network deployment have been proposed. BBU placement strategies based on ILP and heuristic were proposed in [25], where the authors aimed at minimizing the cost for BBUs and fibers by placing BBU sites only in selected buildings. However, in this strategy BBUs are not fully centralized in hotels, which may lead to coordination problems with macro BSs in the area. Optimal placement of RRUs was not addressed in this work.

Strategies for cost-efficient RRU placement for CRA were initially presented in [12], [26]. An ILP formulation of the RRU placement problem with the objective of minimizing the number of RRU cabinets and the total number of RRU units was presented in [12]. To find solutions for larger size problems which cannot be solved by ILP, two heuristic algorithms were proposed in [26]. The algorithms try to enforce RRU cabinet sharing among antennas placed in different buildings, thus minimizing the number of cabinets. Results showed that bringing fiber to every building (i.e., the way conventional deployment approaches work), is the most expensive CRA deployment strategy. In this paper, we extend our preliminary works from [12], [26] by proposing a more general strategy for the minimization of CRA-based network deployment cost.

\section{CRA EnAbling Technology And Planning PROBLEM}

\section{A. Centralized Radio Architecture}

CRA is composed of three main blocks: indoor antennas, remote radio units (RRUs), and baseband units (BBUs). An example of a CRA is depicted in Fig. 1.

Indoor antennas are ultra-compact and provide highcapacity wireless access to a relatively large indoor area (i.e., 500 to $800 \mathrm{~m}^{2}$ ). Indoor antennas are equipped with a small power amplifier, a radiofrequency (RF) filter and a frequency down-/up-converter. Each antenna can perform

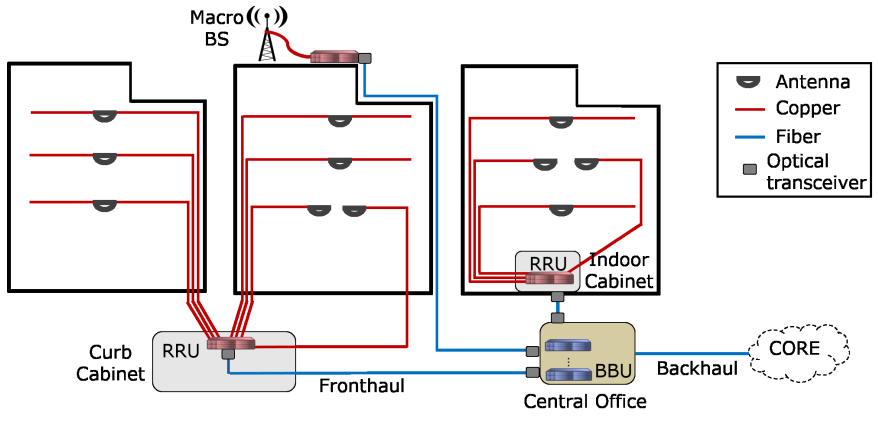

Fig. 1. The Centralized Radio Architecture (CRA) concept. Indoor antennas are connected through copper cables (one for each antenna) to RRUs placed in curb or indoor cabinets. RRUs are connected through dedicated point to point connection to BBUs placed in a centralized location (e.g., a central office owned by a telco).

multiple-input multiple-output (MIMO) transmission/reception and is connected to a RRU. Up to $k$ antennas can be connected to one RRU, each one using a dedicated copper cable [14]. RRUs perform power amplification and measurements of the radio signals as well as Analog-to-Digital/Digital-to-Analog Conversion (ADC/DAC) of the baseband signals to communicate with BBUs. BBUs are in charge of performing digital baseband processing and all the functionalities of the medium access control (MAC) and upper layers [3]. Multiple BBUs can be aggregated in a single BBU Hotel, usually located in a building owned by a telephone service provider (e.g., central office (CO)). A BBU Hotel normally serves a large number of RRUs and (possibly) macro BSs so a single BBU Hotel may cover a wide area.

Indoor antennas communicate with RRUs via analog transmission over a standard copper cable (e.g., Ethernet cable Cat 5/6/7), allowing for the reuse of the existing copper infrastructure inside a building. Antennas perform filtering and frequency up and down conversion of the radio signals to intermediate frequencies in order to fully exploit the bandwidth available over the copper cables. In this way it is possible to fully utilize the spectrum between direct current (DC) and $400 \mathrm{MHz}$ of a Cat.6a cable [14], which is characterized by low noise floor and cable loss. However, copper cables are subject to high attenuation that limits the maximum length of the links between an antenna and a RRU, typically to a few tens of meters, depending on the category of the copper cable.

RRUs are connected to BBUs through the so called fronthaul links. The fronthaul data are transmitted using either analog or digital radio-over-fiber technology (i.e., A-RoF, or $\mathrm{D}-\mathrm{RoF}$, respectively). The latter is the most popular choice and is based on a standard radio interface referred to as common public radio interface (CPRI). D-RoF transmission with CPRI poses strict latency and capacity constraints on the fronthaul segment. In terms of capacity, CPRI requires a constant bitrate of several $\mathrm{Gb} / \mathrm{s}$ [27]. As a result a dedicated fiber connection is required between a RRU and a BBU. The latency requirement, on the other hand, comes from the radio MAC layer functions. More specifically, the hybrid automatic repeat request (HARQ) mechanism in long term evolution (LTE) networks has a constraint on the maximum round trip time that 

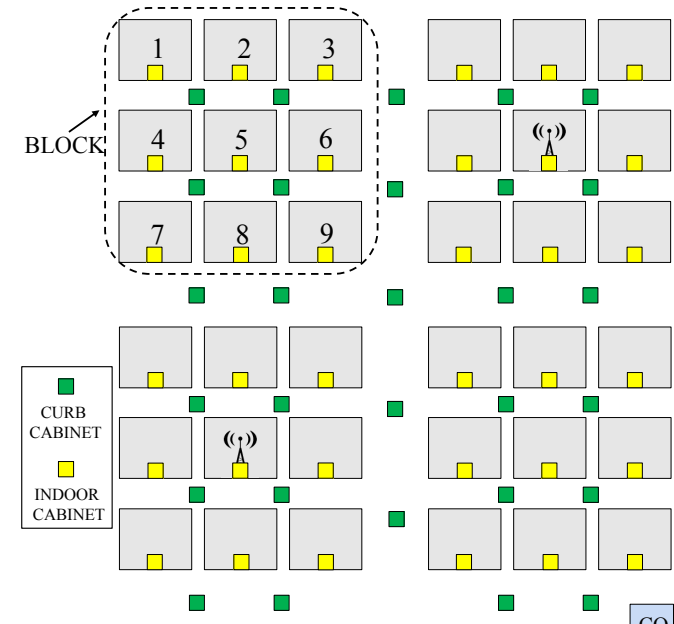

Fig. 2. View from the top of a possible scenario considered in the study.

in turn can be translated into a maximum signal propagation delay over a RRU-BBU link [20], [28]. Considering the speed of light in an optical fiber, this round trip constraint can be transformed into a maximum length of around $20 \mathrm{~km}$ [28]. The distance between RRUs and BBUs determines the amount of fiber cables required in the fronthaul network, which in its turn is translated into the cost for the transport network. Moreover, when MIMO technology is employed, fronthaul capacity requirement is consequently increased. To overcome this problem, a different baseband split can be adopted. In CRAs, some of the baseband functions may be moved from the $\mathrm{BBU}$ to the RRU to realize a different split. It is worth noting that the choice of the baseband split has no effect on antennaRRU link, and does not impact the proposed RRUP strategies. Finally, the segment of the network that connects BBUs with the core of the network is a packet-based traditional backhaul network.

\section{B. CRA Planning Problem Description}

We study the deployment of a CRA in a residential greenfield scenario with no existing network infrastructure other than the copper inside the buildings, e.g., pre-installed Ethernet cables. Figure 2 depicts a schematic view (from the top) of the considered residential area, where the grey squares represent the buildings. We assume that one or more indoor antennas are placed in each floor of every building to provide broadband wireless access to the indoor users. Depending on the distance limitations of the copper links, RRUs can be placed either in indoor cabinets or in curb cabinets located nearby (denoted with the yellow and green squares in Fig. 2, respectively). When inside a building, RRUs are placed at the entrance, where they can be connected to the in-building copper infrastructure. In this case a RRU can be connected only to antennas located in the same building. A RRU placed in a curb cabinet can be connected to antennas in different buildings, which increases the sharing factor of the RRUs, provided that their distances from antennas are shorter than the maximum allowed length of the copper link. We refer to the cabinets that host one or more RRUs as active cabinets.
To serve the outdoor users in the residential area, we assume that macro BSs are placed on the top of some of the buildings. Moreover, a $\mathrm{CO}$ owned by the mobile operator is set up in the area. We assume that all BBUs are placed in the $\mathrm{CO}$ and serve the whole residential area (i.e., all RRUs and macro BSs). This is possible under the assumption that the maximum distance between RRUs, macro BSs, and the $\mathrm{CO}$ is lower than the maximum reach of a fronthaul link (i.e., $20 \mathrm{~km}$ ), which is typically the case in urban scenarios.

In order to calculate the length of fiber/copper, we apply the Taxicab geometry formula, also known as $l_{1}$ norm. For example, given a 3D space and two points $\left(x_{1}, y_{1}, z_{1}\right)$ and $\left(x_{2}, y_{2}, z_{2}\right)$, the length of fiber/copper $d$ needed to connect these two points is computed as:

$$
d=\left|x_{1}-x_{2}\right|+\left|y_{1}-y_{2}\right|+\left|z_{1}-z_{2}\right|
$$

Formula (1) is also used to obtain the distance between an antenna and a RRU located inside a building. The distance between an antenna and a RRU located in a curb cabinet is computed as the sum of: ( $i$ ) the distance from the antenna to the entrance of the building and (ii) the distance from the entrance of the building to the curb cabinet, both computed using (1).

The placement of RRUs directly impacts the overall network cost by affecting: (i) the total length of copper ${ }^{1}$ and fiber cables to be deployed, (ii) the amount of network equipment to buy/operate (i.e., the total number of RRU and BBU units in the network), and (iii) the number of cabinets to activate and manage (i.e., the total number of RRU sites). Therefore, the minimization of the network cost requires judicious policies for solving the cost-minimizing RRU placement problem.

\section{Minimum Cost RRU Placement}

\section{A. Problem Definition}

Given a set of possible RRU locations $R$, the set of antennas $A$ to connect, and the maximum distance $D$ allowed between antennas and RRUs, the objective of the RRU placement problem is to select which cabinets to activate for RRU deployment and which antenna to assign to each RRU such that the total amount of radio resources (i.e., number of RRU and active cabinets) is minimized. A solution must guarantee that each antenna is connected to one RRU while making sure that the distance between any RRU and the antenna it is covering does not exceed $D$.

Possible mappings between antennas and RRUs can be modeled using a binary matrix which we refer to as the coverage matrix $C$. Matrix $C$ is of size $|R| \times|A|$ and contains all possible assignments among RRUs and the antennas within their reach. Every row of $C$ represents a possible RRU location (shown as green and yellow squares in Fig. 2), while every column represents one antenna in the map. An element $C_{i j}$ is set to 1 if the distance between cabinet $i$ and antenna $j$ is less or equal to D, i.e., the antenna can be reached from the cabinet, and to 0 otherwise. Following the antenna-cabinet assignment

\footnotetext{
${ }^{1}$ Since Ethernet cables are assumed to be already deployed inside the buildings, only the copper required to connect the entrance of the buildings to the RRUs placed in curb cabinets is considered.
} 
modelled by matrix $C$, the RRU placement problem can be seen as a variation of the Set Cover Problem (SCP) [29], [30], which is NP-hard [31]. Consequentially, RRUP is also NP-hard, the proof of which can be found in the Appendix. The complexity of this problem makes finding optimal CRA deployment solutions computationally prohibitive and limited to small scenarios, where the number of variables is relatively low.

To solve the RRUP problem, we propose two strategies. The first strategy is based on Integer Linear Program (ILP) and denoted as RRUP-ILP. It is used to obtain optimal solutions for small problem instances [12]. The second strategy, denoted as RRUP-H, is based on a heuristic approach and is capable of finding suboptimal solutions for large network instances within reasonable computational time. Both strategies aim at minimizing the number of RRUs and cabinets to be installed, which dominates the total deployment cost of the network, as supported by the data reported in the results section. For this reason, copper and fiber cables are not included in the minimization.

\section{B. ILP formulation for the RRU placement problem (RRUP- ILP)}

In the following, the ILP formulation of the RRU placement problem is reported. First the notation, input parameters, and decision variables are introduced. Then the objective function and constraints of the problem are reported and explained in detail.

Notation:

- $R$ : set of possible RRU cabinets; each cabinet can host 1 or more RRUs.

- $A$ : set of antenna locations.

- $D$ : maximum allowable distance between a RRU and an antenna.

- $d_{i j}$ : distance between a candidate RRU cabinet $i \in R$ and an antenna $j \in A$.

Input parameters:

- $C[|R| \times|A|]$ : coverage matrix, where $C_{i j}=1$ if a RRU placed in cabinet $i \in R$ can cover antenna $j \in A$, i.e., if $d_{i j} \leq D, 0$ otherwise.

- $M \in \mathbb{N}$ : a large number (e.g., 10000).

- $\alpha, \beta \in \mathbb{N}$ : tuning parameters.

- $k \in \mathbb{N}$ : maximum number of antennas that can be connected to a RRU.

Decision variables:

- $m_{i j} \in\{0,1\}=1$ if a RRU placed in cabinet $i \in R$ is covering antenna $j \in A$; 0 otherwise.

- $r_{i} \in \mathbb{N}=$ the number of RRUs placed in cabinet $i \in R$.

- $z_{i} \in\{0,1\}=1$ if at least one RRU is placed in cabinet $i \in R$; 0 otherwise.

The RRU placement problem is formulated as follows:

$$
\text { Minimize } \alpha \cdot \sum_{i \in R} r_{i}+\beta \cdot \sum_{i \in R} z_{i}
$$

The objective of RRUP-ILP, modelled by the objective function (2), is to minimize the total number of RRU cabinets to be activated and the total number of RRUs to be deployed. Parameters $\alpha, \beta$ balance the contributions of the two components of the objective function.

Subject to the following constraints:

$$
\begin{gathered}
\sum_{i \in R} C_{i j} m_{i j}=1, \forall j \in A \\
k \cdot r_{i} \geq \sum_{j \in A} C_{i j} m_{i j}, \forall i \in R \\
M \cdot z_{i} \geq r_{i}, \forall i \in R \\
r_{i} \geq 0, \forall i \in R
\end{gathered}
$$

Constraint (3) guarantees that each antenna in the network is covered by a RRU within the reach. Constraint (4) ensures that the RRUs placed in cabinet $i$ cover all antennas which are assigned to that cabinet. Constraint (5) models the deployment of RRUs in cabinet $i$ by marking only the locations that host the selected RRUs as active. Finally, constraint (6) guarantees the feasibility of the solution.

\section{Heuristic approach for RRU placement problem (RRUP-H)}

Due to the complexity of the RRU placement problem, finding optimal solutions using RRUP-ILP is limited to small scenarios covering small areas with a low number of users. To deal with CRA network deployment in large residential scenarios, a computationally simpler solution is needed. To address this issue, we develop a scalable two-step heuristic approach for RRU placement, denoted as RRUP-H. RRUP$\mathrm{H}$ aims at minimizing the number of active cabinets and RRUs needed to cover the area in two subsequent phases. The first phase, referred to as Minimum Location Search (MLS), minimizes the set of RRU cabinets that an operator needs to activate in order to serve the area. The second phase, referred to as Radio Unit Minimization (RUM), minimizes the number of RRUs to be placed in the cabinets selected after running MLS.

The pseudocode of the MLS phase is shown in Algorithm 1. The basic idea behind the approach is to increase the chances of sharing the cabinet locations among multiple antennas by assigning blocks of consecutive antennas to the furthest possible RRU location that satisfies the reach constraint. In the initialization phase, the antennas that belong to the same building block are numbered in sequential order starting from the top left corner of the area, as shown in Fig. 2. In addition to the notation used by RRUP-ILP, the MLS phase uses the following sets:

- $V$ : for each cabinet $i, V_{i}$ contains the index of the first unassigned antenna that cannot be connected to $i$ due to the limit on $D$.

- $C S$ : the solution of MLS, containing all the cabinets used by the algorithm (i.e., RRU placement).

The MLS algorithm is executed iteratively until all antennas are covered, i.e., as long as there are non-zero elements in matrix $C$ (line 5). It starts from the lowest-indexed unconnected antenna $j$, represented by the first column in $C$ containing nonzero elements (line 6). It then searches for the RRU cabinet 


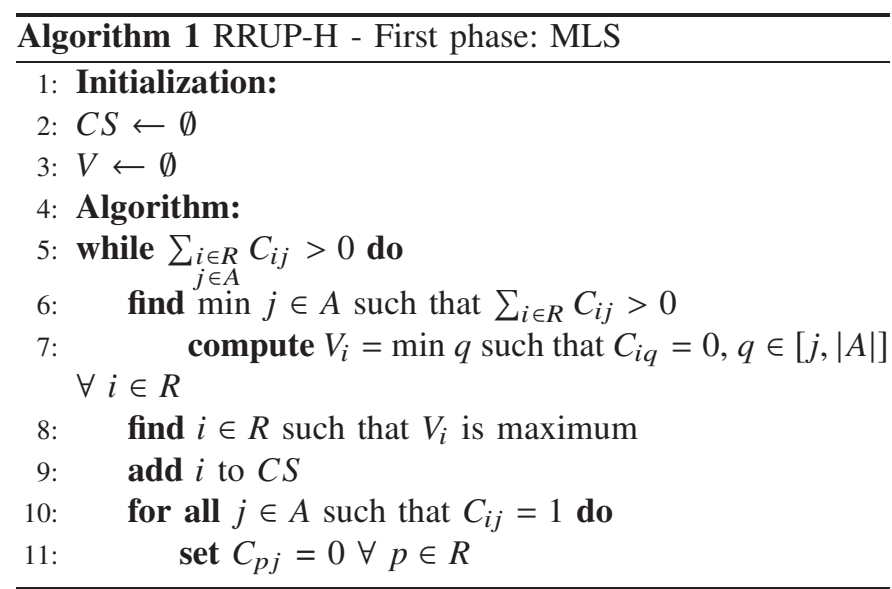

that covers antenna $j$ and the maximum number of consecutive antennas $q, q>j$. For each cabinet $i$, the value of $V_{i}$ is set as the lowest antenna index $q, q>j$ for which $C_{i q}$ is 0 (line 7). The algorithm then selects the RRU location $i$ with the maximum value of $V_{i}$, i.e., the cabinet which covers the largest number of consecutive antennas (line 8). As only blocks of consecutive ones are considered, selecting cabinet $i$ guarantees that no antennas are left disconnected after making this choice. The selected cabinet is added to CS (line 9) and all antennas within its reach are removed from matrix $C$ by changing the values of elements $C_{i j}$ from 1 to 0 in all corresponding columns (lines 10 - 11).

The set of cabinets $C S$ obtained by MLS is passed to the Radio Unit Minimization (RUM) phase, aimed at minimizing the number of RRUs placed at the cabinets to cover the area. The idea is to obtain the utilization of RRU ports as close to $100 \%$ as possible, in order to reduce the number of unconnected ports and, consequently, the waste of RRU resources. To model the antenna-RRU assignment, RUM uses the following additional structures:

- $C^{\prime}$ : updated coverage matrix obtained from $C$ by considering only the cabinets included in the solution $C S$. Element $C_{i j}^{\prime}$ is equal to 1 if a RRU placed at a selected cabinet $i$ can cover antenna $j$, and to 0 if it is out of reach.

- $A C$ : the final solution of RRUP-H, containing the assignment of antennas to cabinets. Each $A C_{i}$ contains the set of antennas assigned to cabinet $i$.

The pseudocode of the RUM phase is shown in Algorithm 2. It assigns antennas to cabinets and places RRUs at cabinet locations in four steps. The first step (lines 4 - 7) performs assignment of antennas that can be linked to only one cabinet, making sure that RRUs are placed at these indispensable locations. The algorithm identifies such antennas as those whose corresponding columns in $C$ have only a single nonzero element (line 4), and determines their matching cabinets (line 5). The assigned antennas are added to the solution set $A C$ (line 6) and matrix $C^{\prime}$ is updated by deleting these antennas (line 7). The second step of RUM (lines 8 - 12) tries to maximize the utilization of the RRUs deployed in step 1 by using up as many of their ports as possible. To do so, the algorithm identifies cabinets that host RRUs with free ports

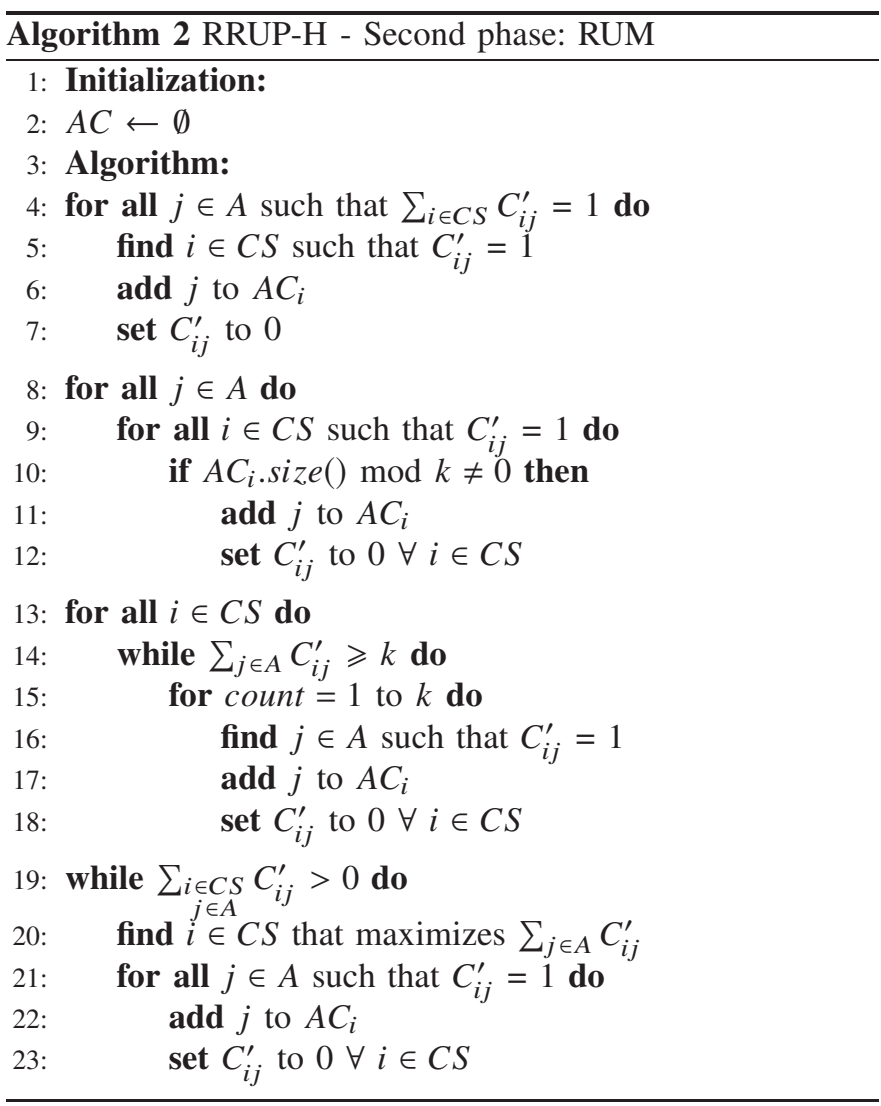

(line 10), assigns to them antennas within reach (line 11), and updates matrix $C^{\prime}$ to reflect this assignment (line 12). The third step of RUM deploys additional RRUs that can be fully utilized by the unconnected antennas (lines $13-18$ ). For each of the cabinets contained in $C S$, the algorithm checks whether they can be connected to $k$ antennas (lines 13 and 14). Groups of $k$ antennas are iteratively connected to selected locations (lines 15 - 18) by adding each antenna to the chosen cabinet (line 17) and updating the $C^{\prime}$ matrix (line 18). Finally, the fourth step of RUM connects the remaining antennas (lines 19 - 23). In this phase, the algorithm starts from the cabinet capable of connecting to the highest number of unconnected antennas, identified by the row of matrix $C^{\prime}$ with the greatest number of ones (line 20). Each antenna which can be connected to that cabinet (see condition in line 21) is assigned to it (line 22) and matrix $C^{\prime}$ is updated (line 23). This is the final phase of the RUM algorithm, which ends when all antennas are covered (line 19).

\section{Illustrative Example}

To show the benefits of our approach we compare it with a conventional deployment approach called Radio over Fiber To the Building (RTB) [32]. This approach places RRUs only at the building entrances (denoted with yellow squares in Fig. 2) and connects them to the BBU Hotels with a dedicated fiber. Since RTB does not place RRUs in curb cabinets, each RRU covers only the antennas inside the building in which it is located.

Figure 3 shows a simple example illustrating the operation principles and the solutions obtained by RTB (Fig. 3a) and 


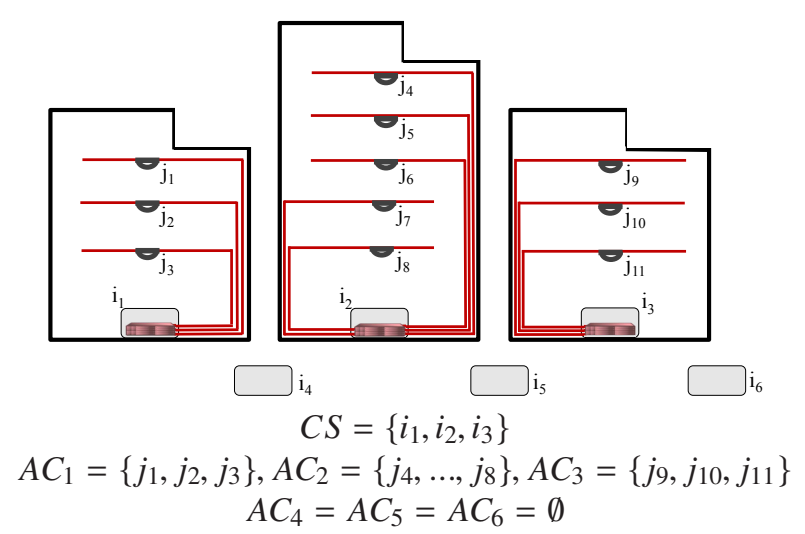

(a) RTB based design.

Fig. 3. Example of network deployment using RTB and RRUP-H.

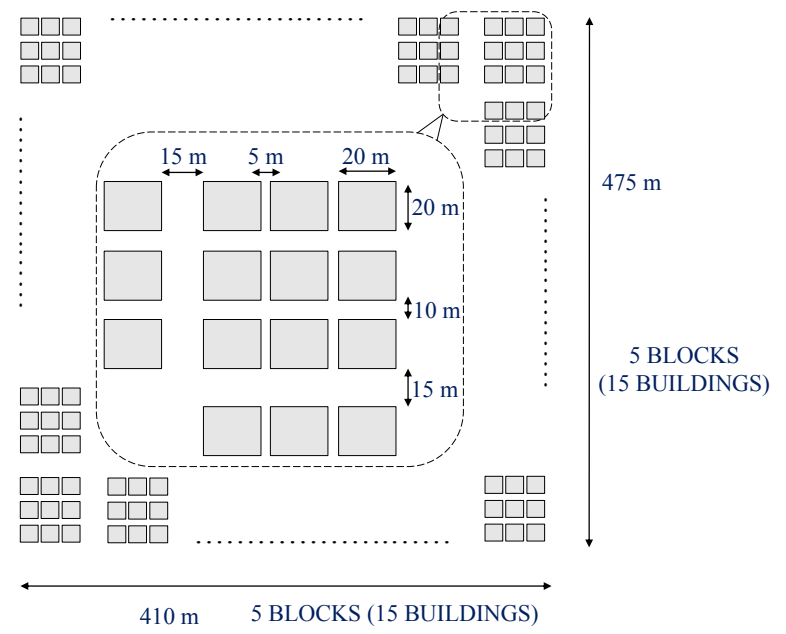

Fig. 4. An example of a residential district simulation scenario showing the size.

RRUP-H (Fig. 3b). From the figures it can be observed that, in the RTB case, three cabinets are activated to host RRUs $\left(i_{1}, i_{2}, i_{3}\right)$. Considering that up to 8 antennas can be connected to a single RRU [14], three RRUs are required. On the other hand, RRUP-H, which maximizes the sharing of RRUs among different buildings, requires only one active cabinet $\left(i_{5}\right)$ and two RRUs, decreasing the amount of components to be deployed and lowering the associated costs.

\section{Numerical Results}

The performance of the RRUP-ILP and RRUP-H approaches is evaluated via simulations in two deployment scenarios. We first evaluate the efficiency of RRUP-H as a good solution to the RRUP problem by comparing it to the optimal solutions obtained by RRUP-ILP in a small-sized scenario denoted as Residential District. We then evaluate the savings attainable by RRUP-H in a full-sized realistic network scenario with various positions and numbers of BBU Hotels, denoted as Urban. Optimal solutions are obtained by using the commercially available solver CPLEX [33], run on a HP workstation with a $2.67 \mathrm{GHZ}$ processor and $16 \mathrm{~GB}$ RAM.

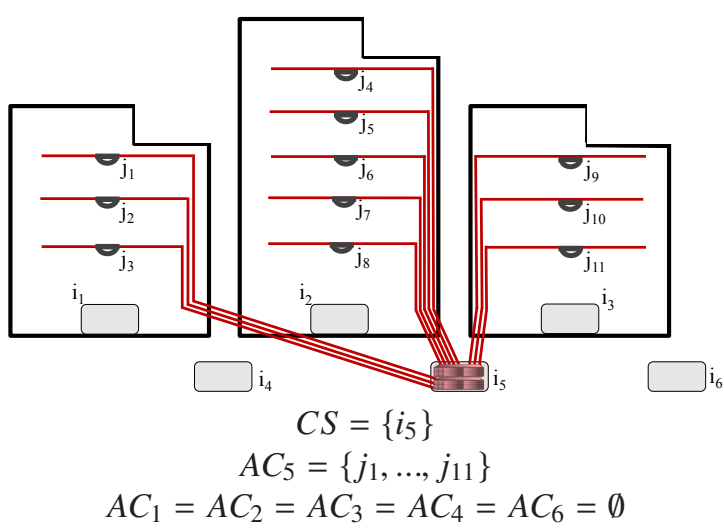

(b) RRUP-H based design.

\section{A. Residential District Scenario}

The Residential District scenario is based on a Manhattan street model with buildings arranged in blocks, as depicted in Fig. 4. The considered area is composed of 25 blocks organized in a $5 \times 5$ matrix. The total size of the map is $410 \times 475 \mathrm{~m}^{2}$. Each block is composed of 9 buildings organized in a $3 \times 3$ matrix and the blocks are divided by $15 \mathrm{~m}$ wide streets. Buildings within a block are separated by $10 \mathrm{~m}$ wide horizontal streets and $5 \mathrm{~m}$ wide vertical streets. Each building is represented by a square with $20 \mathrm{~m}$ sides, while the height of each floor is $3 \mathrm{~m}$. The number of floors in each building is a random variable following a discrete uniform distribution over the interval $[1,12]$ and the results are averaged over 10 different configurations of the scenario. In our case study, we assume that one omnidirectional indoor antenna is placed in the center of the ceiling on each floor and is sufficient to cover the entire floor. Each antenna is connected to a RRU through a Category 6 copper cable and each RRU is connected to a BBU port through a dedicated fiber. Each point-to-point fiber link between a RRU and a BBU requires two enhanced small form pluggable (SFP+) optical transceivers. We assume that an operator, to serve the outdoor users, has already deployed two macro BSs in the area. These BSs are connected to the $\mathrm{CO}$ hosting their assigned BBUs through dedicated optical fibers. The limited size of this scenario allows placing all BBUs in the $\mathrm{CO}$ that is located in the right bottom corner of the map [12].

To evaluate the impact of the maximum link length $(D)$ between RRUs and antennas, in the simulations we set the value of $D$ to $50 \mathrm{~m}, 75 \mathrm{~m}$ and $100 \mathrm{~m}$, the latter corresponding to the maximum distance over a twisted pair cable in the 1000 BASE-T standard. The values for $\alpha$ and $\beta$ were determined via a sensitivity analysis, whose results are reported in Table I for the case $D=50 \mathrm{~m}$, and $D=100 \mathrm{~m}$. The table shows that, for the case $D=50 \mathrm{~m}$, when $\alpha>>\beta$ the number of RRUs decreases, at the expense of a higher number of RRU cabinets. Conversely, when $\beta>>\alpha$ the number of RRU cabinets is reduced, but a larger number of RRUs is required. For the case $D=100 \mathrm{~m}$ no changes are experienced, therefore the choice of the values of $\alpha$ and $\beta$ has no impact on the number of RRUs and cabinets. Since the cost of a cabinet is higher 


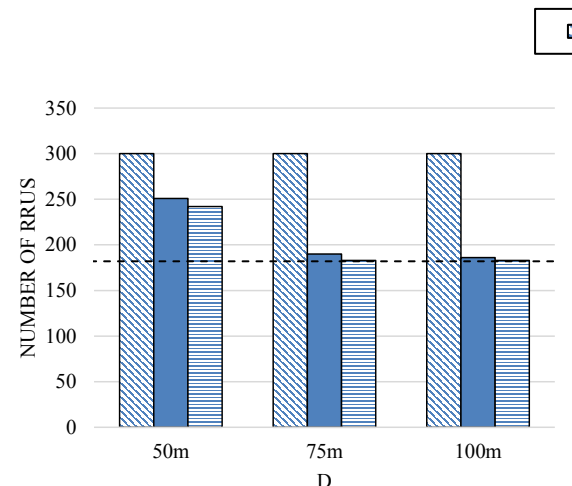

(a)

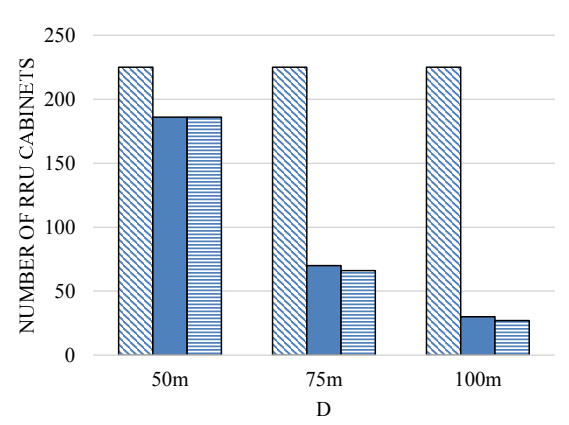

(b)

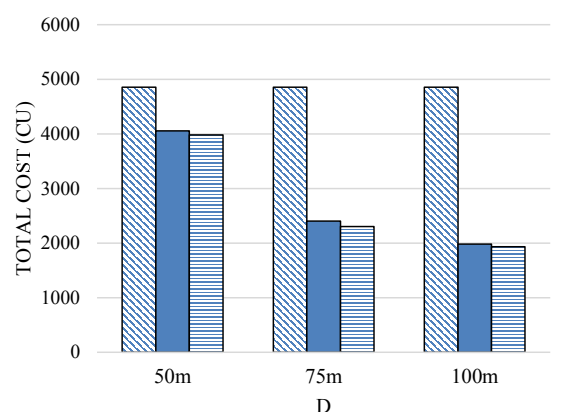

(c)

Fig. 5. The number of RRUs (a), RRU cabinets (b) that needs to be activated and total cost of the network (c) as a function of $D$, the maximum distance between antennas and RRU, in residential district scenario.

TABLE I

IMPACT OF DIFFERENT VALUES OF $\alpha$ AND $\beta$ ON THE SOLUTIONS OBTAINED BY RRUP-ILP FOR D=50 $\mathrm{m}$ AND D=100 $\mathrm{m}$.

\begin{tabular}{cccccc}
\hline \hline \multicolumn{7}{c}{$\mathbf{D = 5 0} \mathbf{~ m}$} \\
\hline$(\alpha, \beta)$ & $(10,1)$ & $(2,1)$ & $(1,1)$ & $(1,2)$ & $(1,10)$ \\
\# RRU cabinets & 203 & 203 & 198 & 186 & 184 \\
\# RRUs & 228 & 228 & 233 & 245 & 249 \\
\hline \multicolumn{7}{c}{$\mathbf{D = 1 0 0} \mathbf{~ m}$} \\
\hline$(\alpha, \beta)$ & $(10,1)$ & $(2,1)$ & $(1,1)$ & $(1,2)$ & $(1,10)$ \\
\# RRU cabinets & 26 & 26 & 26 & 26 & 26 \\
\# RRUs & 186 & 186 & 186 & 186 & 186 \\
\hline \hline
\end{tabular}

TABLE II

AVERAGE SOLVING TIME REQUIRED BY RRUP-ILP AND RRUP-H FOR DIFFERENT VALUES OF ANTENNA-RRU LINKS (D).

\begin{tabular}{ccc}
\hline \hline Distance ant-RRU & RRUP-ILP time & RRUP-H time \\
$50 \mathrm{~m}$ & $7.7 \mathrm{~s}$ & $<100 \mathrm{~ms}$ \\
$75 \mathrm{~m}$ & $19.7 \mathrm{~s}$ & $<100 \mathrm{~ms}$ \\
$100 \mathrm{~m}$ & $30 \mathrm{~min}$. & $<100 \mathrm{~ms}$ \\
\hline \hline
\end{tabular}

than the cost of a RRU, we chose to use $\alpha=1$ and $\beta=2$. In all scenarios, the value of $k$ was set to 8 [14] while the maximum number of RRUs that can be connected to a single BBU was set to 6 [14]. Table II reports the running times of RRUP-ILP and RRUP-H for different values of D. The ILP can be solved in half an hour for D equal to $100 \mathrm{~m}$, which can be acceptable for a network planning problem. The heuristic strategy takes considerably lower running time to find sub-optimal solutions with acceptable quality.

Figure 5a shows the number of RRUs required by the RRUP-ILP, RRUP-H and RTB approaches to serve the area as a function of $D$. The figure also includes a theoretical lower bound (LB) on the number of RRUs that would be required to cover the area without any limitation on the length of the copper links (i.e., for $D=\infty$ ), allowing for full RRU centralization at a single site. It can be observed that RRUP-H and RRUP-ILP achieve a significant reduction in the number of RRUs compared to the conventional RTB approach. In general, RRUP-H performs very closely to RRUP-ILP, deploying only $3 \%$ more RRUs on average over all test instances. While the amount of radio equipment required by RTB and LB remains
TABLE III

THE TOTAL LENGTH OF COPPER AND FIBER LINKS IN THE RESIDENTIAL DISTRICT SCENARIO.

\begin{tabular}{ccc}
\hline \hline Algorithm & $\begin{array}{c}\text { Copper } \\
\text { cable }(\mathbf{k m})\end{array}$ & $\begin{array}{c}\text { Fiber } \\
\text { cable }(\mathbf{k m})\end{array}$ \\
\hline RRUP-ILP 50m & 45.1 & 106.4 \\
RRUP-H 50m & 44.9 & 110.0 \\
RRUP-ILP 75m & 82.0 & 79.9 \\
RRUP-H 75m & 83.6 & 82.5 \\
RRUP-ILP 100m & 102.3 & 80.3 \\
RRUP-H 100m & 102.9 & 80.6 \\
RTB & 34.9 & 132.0 \\
\hline \hline
\end{tabular}

the same for different values of $D$, RRUP-ILP and RRUP$\mathrm{H}$ reduce the equipment volume for longer reach values. In particular, RRUP-H uses $16 \%, 37 \%$, and $38 \%$ less units than RTB in case of $D=50 \mathrm{~m}, D=75 \mathrm{~m}$, and $D=100 \mathrm{~m}$, respectively. In addition, for medium and higher reach, RRUP$\mathrm{H}$ performs very close to the $\mathrm{LB}$ and uses only $4 \%$, and $2 \%$ more RRUs than LB for $D$ equal to 75 and $100 \mathrm{~m}$, respectively. Since all BBUs are placed in the same BBU Hotel, the number of BBUs required by the different strategies is obtained by dividing the number of RRUs by the maximum number of RRUs that can be connected to a single BBU (fixed and equal to 6).

Figure $5 \mathrm{~b}$ shows the number of cabinets to be activated by an operator. Similarly to the previous figures, the number of active cabinets obtained by RRUP-H is almost the same as for RRUP-ILP. The number of cabinets required by RRUP-ILP and RRUP-H decreases as $D$ increases, and for $\mathrm{D}$ equal to $100 \mathrm{~m}$ it is by one order of magnitude lower than the number obtained by RTB. For LB the number of required cabinets is always equal to 1 (omitted from the graph), since $D=\infty$ allows to place all RRUs in the same location.

Table III reports the total length of copper cables and optical fibers computed by RRUP-ILP, RRUP-H and RTB. It can be observed that higher values of $D$ allow for greater RRU aggregation and larger distances between RRUs and antennas, thus increasing the length of copper cables and decreasing the length of optical fibers. For RTB, the amount of equipment and active cabinets is constant, so the length of cables does not change with $D$. 
TABLE IV

THE NORMALIZED COST OF THE NETWORK COMPONENTS [34] [35].

\begin{tabular}{cc}
\hline \hline Component & Normalized cost $(\mathbf{C U})$ \\
\hline SFP+ & 1 \\
RRU & 3.75 \\
BBU & 15 \\
RRU Cabinet & 10 \\
BBU Cabinet & 20 \\
Copper cable (Cat. 6) $(\mathrm{km})$ & 1 \\
Multi-modal fiber cable $(\mathrm{km})$ & 1 \\
\hline \hline
\end{tabular}

TABLE V

THE NUMBER OF RRUS, RRU CABINETS, AND COPPER CABLES REQUIRED BY EACH ALGORITHM FOR THE URBAN SCENARIO.

\begin{tabular}{cccc}
\hline \hline Algorithm & RRUs & $\begin{array}{c}\text { RRU } \\
\text { cabinets }\end{array}$ & $\begin{array}{c}\text { Copper } \\
\text { cables (km) }\end{array}$ \\
\hline RRUP-H 50m & 31387 & 23175 & 1317.0 \\
RRUP-H 75m & 23585 & 8619 & 6269.9 \\
RRUP-H 100m & 23343 & 3525 & 8778.3 \\
RTB & 37605 & 28224 & 0 \\
LB & 22904 & 1 & N.A. \\
\hline \hline
\end{tabular}

Figure $5 \mathrm{c}$ shows the capital expenditure values for each approach, calculated as a sum of the normalized cost of each component reported in Table IV. The values in the figure refer to the equipment, cables and cabinets to be deployed in each solution, without considering the cost for the pre-installed copper infrastructure inside buildings. As the equipment deployed by RTB does not depend on the values of $D$, its total cost is also constant. The costs of the RRUP-ILP and RRUP-H solutions decrease for greater values of $D$, i.e., with a longer reach less active cabinets and RRUs are needed. When $D$ equals $100 \mathrm{~m}$, RRUP-ILP and RRUP-H reduce the cost by $59 \%$ with respect to RTB. In addition, they show very similar performance, with RRUP-H yielding only $2 \%$ higher costs than RRUP-ILP.

\section{B. Urban Scenario}

In the Urban Scenario, also based on a Manhattan grid, the map is composed of 3136 blocks organized in a $56 \times 56$ matrix for a total of 28224 buildings. The total size of the map is $4745 \times 5320 \mathrm{~m}^{2}$, i.e., $25 \mathrm{~km}^{2}$, which can be considered as the urban area of a medium-sized European city. The number of buildings per block, the street dimensions and all other parameters match the values used in the Residential District scenario. We run the simulations for values of $D$ equal to 50 , 75 , and $100 \mathrm{~m}$.

Table V reports the amount of the RRUs, RRU cabinets and copper cables required to cover the area with RRUP-H and RTB, as well as the value of LB. Ethernet cables are assumed to be already deployed in the buildings, thus the cost for copper cables in the RTB case, where RRUs can be placed only inside a building, is 0 . By comparing the solutions obtained with RRUP-H and RTB, it is possible to observe that the number of RRUs required by RRUP-H is $16 \%, 37 \%$, and $38 \%$ lower than the RTB case for $D=50 \mathrm{~m}, D=75 \mathrm{~m}$, and $D=100 \mathrm{~m}$, respectively. Moreover, RRUP-H requires $37 \%$, $3 \%$, and $2 \%$ more RRUs than LB for the considered values

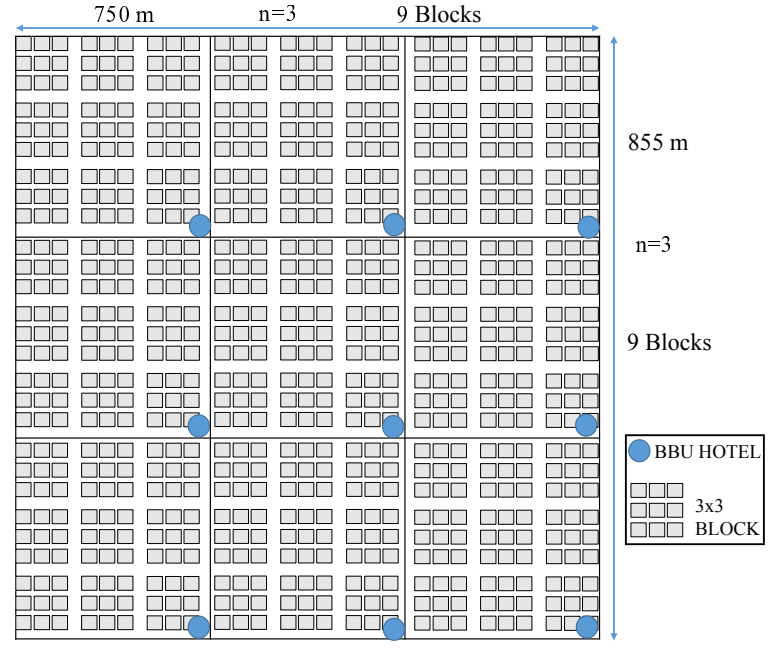

Fig. 6. An example of a BBU Hotel placement for $n=3$.

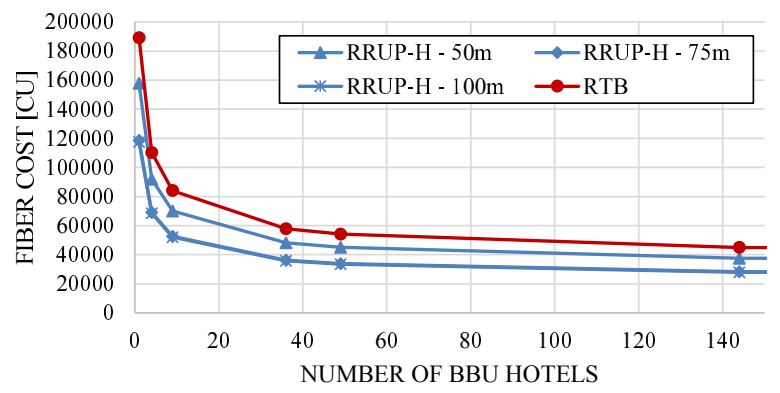

Fig. 7. The fiber cost for RTB and RRUP-H, for the three values of $D$, as a function of the number of BBU Hotels in the urban scenario.

of $D$. These RRU usage values, which are very close to the ones reported for the Residential District scenario, confirm the effectiveness of our heuristic and demonstrate its capability of reaching superior results also in large scenarios.

To investigate the impact of the number of BBU Hotels on the overall solution, we tune the density of their locations by dividing the area into $n \times n$ sub-areas, each one equipped with a BBU Hotel. In the simulations, the set of values assumed by $n$ is a subset of the divisors of $168,\{1,2,3,6,7,12,42\}$. To clarify this partition, an example with $n=3$ and 81 blocks is depicted in Fig. 6. Each Hotel is placed in the bottom right corner of a sub-area (blue circles in Fig. 6) and all RRUs in that sub-area connect to it. Each BBU is connected to the CO via an optical grey point-to-point link and two SFP+. The CO performs coordination functions (e.g., CoMP), and is located in the bottom right corner of the map.

Figure 7 shows the fiber cost as a function of the number of BBU Hotels and different values of $D$. The reported costs include both the fibers used in the fronthaul link and the fibers that carry the aggregated traffic from the BBUs to the CO. It can be observed from the figure that the RRUP$\mathrm{H}$ approach obtains solutions with lower cost than that of RTB in all test cases. For the most constrained reach of $D=50 \mathrm{~m}$, the RRUP-H obtains $16 \%$ lower cost than RTB, while the savings for $D=75$ and $100 \mathrm{~m}$ equal respectively $37 \%$ and $38 \%$ on average over all considered BBU Hotel 


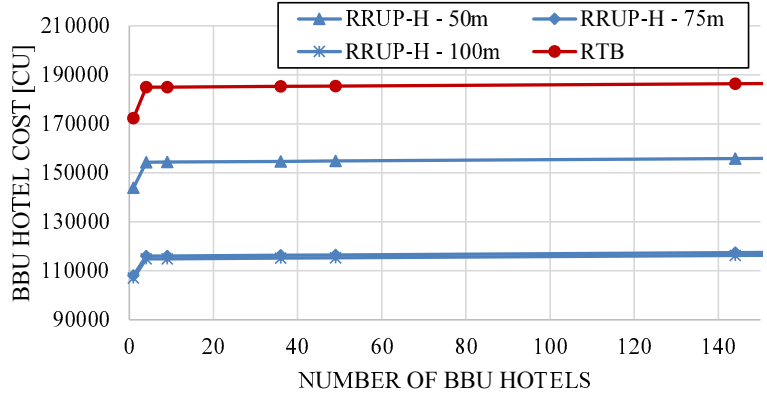

Fig. 8. The sum of the costs related to BBUs, BBU cabinets, and SFP+ as a function of the number of BBU Hotels in the urban scenario.

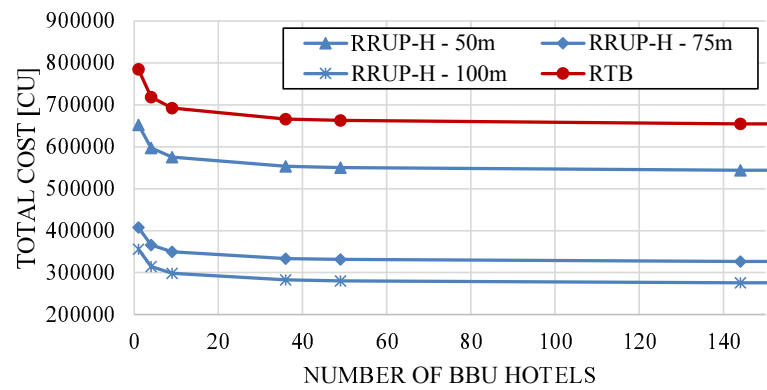

Fig. 9. The total cost of RTB and RRUP-H, for the three values of $D$, as a function of the number of BBU Hotels in the urban scenario.

number values. Moreover, the total fiber cost values drop exponentially with increasing numbers of BBU Hotels. This is due to the fact that a higher number of Hotels allows for placing BBUs closer to RRUs, thus achieving a consistent reduction of the fronthaul link lengths. However, increasing the number of BBU Hotels also increases the number of BBUs, BBU cabinets and SFP+, aggregately referred to as BBU Hotels cost. Figure 8 reports the BBU Hotels cost as a function of the number of BBU Hotels. The figure shows that the BBU Hotels cost increases linearly with the number of Hotels. Again, RRUP-H outperforms RTB, yielding 16\%, $37 \%$, and $38 \%$ lower costs than RTB for the $D=50,75$, and $100 \mathrm{~m}$ case, respectively. These results show that the RRUs minimization introduced by RRUP-H has a direct impact on the cost of the network. In particular, a RRUs reduction of $16 \%, 37 \%$, and $38 \%$ translates into a reduction of $16 \%, 37 \%$, and $38 \%$ of both BBU Hotels and fiber cost.

Figure 9 shows the total deployment costs, which account for the cost of each CRA component, as a function of the number of BBU Hotels and different values of $D$. It can be observed that, for each value of $D$, the cost drops significantly when the number of BBU hotels increases. This is because with $144 \mathrm{BBU}$ Hotels the costs are dominated by the fiber deployment cost. However, increasing the number of BBU Hotels leads to a modest growth (not reported in the graph for the sake of clarity) of the total cost because the cost of the BBU Hotels becomes dominant, (i.e., a higher number of BBU Hotels implies shorter fronthaul distances). This fact is highlighted in Table VI, which reports the exact values of the cost obtained by each algorithm for three different numbers of Hotels. The table indicates that the solutions found by RRUP$\mathrm{H}$ for $D=100 \mathrm{~m}$ with 9 and 1764 Hotels are $7.6 \%$ and $5.3 \%$
TABLE VI

TOTAL COST [CU] WITH 9, 144, AND 1764 HOTELS FOR EACH ALGORITHM IN THE URBAN SCENARIO.

\begin{tabular}{cccc}
\hline \hline & $\mathbf{9}$ & $\mathbf{1 4 4}$ & $\mathbf{1 7 6 4}$ \\
Algorithm & Hotels & Hotels & Hotels \\
\hline RRUP-H 50m & 575272 & 544089 & 557529 \\
RRUP-H 75m & 349594 & 326565 & 341685 \\
RRUP-H 100m & 298413 & 275738 & 291262 \\
RTB & 692300 & 654596 & 665477 \\
\hline \hline
\end{tabular}

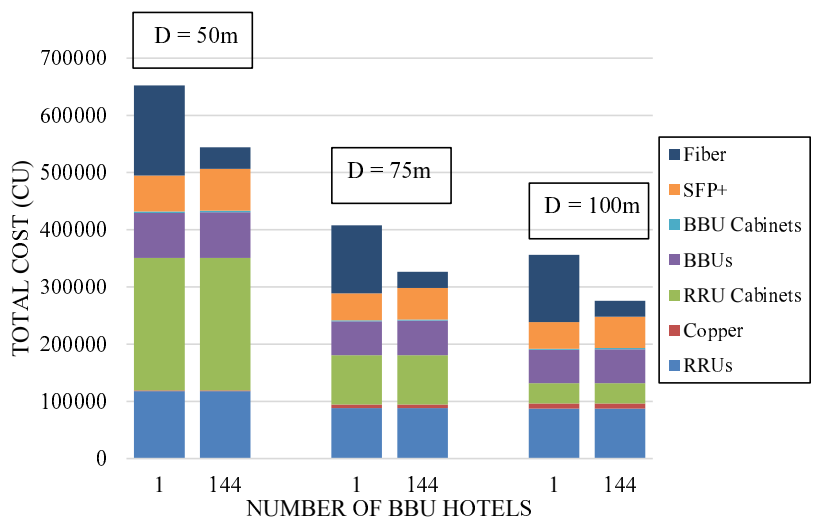

Fig. 10. The contribution of each network component to the total cost of RRUP-H and RTB as a function of the number of BBU Hotels in the urban scenario.

more expensive than the one with 144 Hotels, respectively. Therefore, from an equipment cost perspective, 144 Hotels can be considered as the best solution. On the one hand, using 144 Hotels may lead to excessive operational costs, while on the other hand, reducing the number of Hotels by centralizing a large amount of BBUs in the same site may increase the computational resources required for an efficient interference management. This aspect is out of scope of this paper and requires additional studies.

Figure 10 depicts the contributions of each network component to the total cost of the RRUP-H solutions for each value of $D$, and for two amounts of BBU Hotels, i.e., 1 and 144. The figure shows that increasing the number of Hotels to 144 allows for a total cost reduction of $22 \%$ for $D=100 \mathrm{~m}$. Moreover, it can be noticed that the variation of the number of Hotels has no effect on the cost contribution from RRUs, RRU cabinets, and copper cables. On the other hand, the cost contributions due to the amount of fiber, BBUs, BBU cabinets, and SFP+ change with different settings.

\section{CONCLUSION}

This paper investigated the possibility to provide enhanced capacity to indoor users by means of CRA networks, from a deployment cost perspective. Thanks to baseband function centralization, CRA allows for using different functional splits, making this architectural solution extremely flexible and future proof towards 5G. In this paper, the RRU placement (RRUP) problem is defined and proved to be NP-hard, which translates into high complexity while solving the CRA network deployment optimization problem. To find optimal solutions for small problem instances, the RRUP problem is formulated as an ILP 
with the objective of minimizing ( $i$ ) the number of active sites in a residential area that host RRUs and (ii) the total number of deployed RRUs. To address the computational complexity of the ILP approach, a scalable and efficient heuristic algorithm suitable for large scenarios is also proposed. Simulation results show that by enabling sharing of RRUs both approaches can reduce the amount of required radio equipment and active cabinets, which represent the major contribution to the network cost. Consequently, the proposed strategies are able to achieve almost $60 \%$ of cost savings with respect to a conventional approach based on radio over fiber to the building. In addition, results show that increasing the number of active BBU Hotels reduces the overall amount of fibers required, which translates into an additional $22 \%$ of cost savings.

\section{APPENDIX}

In order to prove NP-hardness of the RRUP problem, we first decompose it into two sub-problems, denoted as RRUP $_{C A B}$ and RRUP $R R U$. RRUP ${ }_{C A B}$ refers to the problem of finding a minimum number of cabinets to host the RRUs, while RRUP $_{R R U}$ refers to the placement of a minimum number of RRUs at the locations selected by RRUP ${ }_{C A B}$. In this section, we derive the complexity of the RRUP problem by proving that the RRUP RAB sub-problem is NP-hard.

RRUP $_{C A B}$ takes as input a set of antennas $A=$ $\left\{a_{1}, a_{2}, \ldots, a_{n}\right\}$ and a collection of possible cabinet locations $\mathcal{R}=\left\{R_{1}, R_{2}, \ldots, R_{m}\right\}$, where $R_{i}$ contains a subset of antennas within reach of cabinet $i$. Every antenna is within reach of at least one cabinet location, i.e., $\bigcup_{i \in \mathcal{R}} R_{i}=A$. The output of the problem is a subset $C \subseteq \mathcal{R}$ with minimum cardinality that covers all antennas, i.e., $\bigcup_{i \in C} R_{i}=A$. We show that RRUP $_{C A B}$ is NP-hard by reducing the well-known Set Cover Problem (SCP) to it. An instance of the SCP is defined by a set of elements $U=\left\{x_{1}, x_{2}, \ldots, x_{p}\right\}$ called universe, and a collection of subsets $\mathcal{S}=\left\{S_{1}, S_{2}, \ldots, S_{q}\right\}$ of the universe such that every element of $U$ belongs to at least one subset in $\mathcal{S}$, i.e., $\bigcup_{i \in \mathcal{S}} S_{i}=U$. The solution of the SCP is a subset $\mathcal{D} \subseteq \mathcal{S}$ with minimum cardinality that guarantees that $\bigcup_{i \in \mathcal{D}} S_{i}=U$.

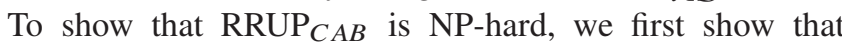
solving the SCP on the same input data of $\operatorname{RRUP}_{C A B}$ would

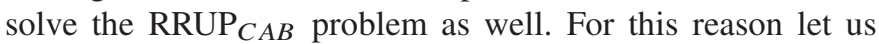
first assume the following instance of the SCP. Let us build set $U$ as the set of antennas to be covered, i.e., each element $u \in U$ corresponds to an element $a \in A$. Let us also assume that each subset $S_{i} \in \mathcal{S}$ defines the subset of antennas $a \in A$ reachable from cabinet location $i$, i.e., by construction set $\mathcal{S}$ and set $\mathcal{R}$ are the same.

If we now assume that $\mathcal{D} \in \mathcal{S}$ is a solution of the SCP of $U$, we can build a set $C \in \mathcal{R}$, where each $S_{i} \in \mathcal{D}$ corresponds to one $R_{i} \in C$. In order for $C$ to represent a solution of RRUP $_{C A B}, C$ must cover all antennas and must be of minimal cardinality. The first statement can be derived from $C=\mathcal{D}$, $\bigcup_{i \in \mathcal{D}} S_{i}=U$, and $U=A$, which imply that $\bigcup_{i \in C} R_{i}=A$. The second statement can be proven by contradiction as follows. Sets $C$ and $\mathcal{D}$ are constructed so that $|C|=|\mathcal{D}|$. Let us assume that there is a subset $\mathcal{F} \subseteq \mathcal{R}$ solving the $\operatorname{RRUP}_{C A B}$ problem such that $|\mathcal{F}|<|C|$. If $\mathcal{F}$ is a solution of $\operatorname{RRUP}_{C A B}$ this means that $\bigcup_{i \in \mathcal{F}} R_{i}=A$, but since $A=U$ and $\mathcal{R}=\mathcal{S}$ this also means that $\mathcal{F}$ is a solution of the $\mathrm{SCP}$ (i.e., $\bigcup_{i \in \mathcal{F}} S_{i}=U$ ), where $|\mathcal{F}|<|\mathcal{D}|$. The last statement contradicts the hypothesis that $\mathcal{D}$ is minimum-sized set cover for $U$.

To complete the proof we need to show that a solution of the RRUP ${ }_{C A B}$ problem is also a solution of the same instance of the SCP, which can be derived in a straightforward way. Let us assume that subset $C \subseteq \mathcal{R}$ solves the $\operatorname{RRUP}_{C A B}$ problem. This implies $\bigcup_{i \in C} R_{i}=A$, but since $A=U$ and $\mathcal{R}=\mathcal{S}$, it also means that $\bigcup_{i \in C} S_{i}=U$, i.e., $C$ is a solution of the SCP. The minimum cardinality of $C$ can be proved by contradiction in the same way as it was done before. Let us now assume that there is a subset $\mathcal{F} \subseteq \mathcal{S}$ solving the SCP such that $|\mathcal{F}|<|C|$. If $\mathcal{F}$ is a solution of SCP this means that $\bigcup_{i \in \mathcal{F}} S_{i}=U$, but since $U=A$ and $\mathcal{S}=\mathcal{R}$ it also means that $\mathcal{F}$ is a solution of the RRUP $_{C A B}$ problem (i.e., $\bigcup_{i \in \mathcal{F}} R_{i}=A$ ), where $|\mathcal{F}|<|C|$. The last statement contradicts the hypothesis that $C$ is minimumsized set that covers all antennas in $A$.

\section{ACKNOWLEDGMENT}

This work has been supported by VINNOVA as part of the project "Smart City Concepts in Curitiba: Innovation for Mobility and Energy Efficiency" and by the European Institute of Innovation and Technology (EIT Digital) project "The Royal Gardens Case".

\section{REFERENCES}

[1] "Ericsson mobility report," Ericsson, Feb. 2016.

[2] N. Al-Falahy and O. Y. Alani, "Technologies for 5G networks: Challenges and opportunities," IT Professional, vol. 19, no. 1, pp. 12-20, Jan 2017.

[3] A. Checko et al., "Cloud RAN for mobile networks - a technology overview," IEEE Communications Surveys Tutorials, vol. 17, no. 1, pp. 405-426, Firstquarter 2015.

[4] M. Fiorani et al., "Energy performance of C-RAN with 5G-NX radio networks and optical transport," 2016 IEEE International Conference on Communications (ICC).

[5] "Project RAN evolution: Further study on critical C-RAN technologies," NGMN, March 2015.

[6] "Small cell virtualization: Functional splits and use cases," Small Cell Forum, Release 7.0, Jan 2016.

[7] "http://www.cpri.info/press.html."

[8] "Ten key rules of 5G deployment: Enabling $1 \mathrm{Tbit} / \mathrm{s} / \mathrm{km} 2$ in 2030," Nokia, 2016.

[9] R. A. Pitaval et al., "Full-duplex self-backhauling for small-cell 5G networks," IEEE Wireless Communications, vol. 22, no. 5, pp. 83-89, October 2015.

[10] T. Okuyama et al., "Antenna deployment for 5G ultra high-density distributed antenna system at low SHF bands," in 2016 IEEE Conference on Standards for Communications and Networking (CSCN), Oct 2016, pp. 1-6.

[11] "Ultra dense network (UDN) white paper," Nokia White paper, 2016.

[12] F. Tonini et al., "Minimum cost deployment of radio and transport resources in centralized radio architectures," Proc. of IEEE International Conference on Computing, Networking and Communications (ICNC) 2016, Feb. 2016.

[13] J. Gambini and U. Spagnolini, "Wireless over cable for femtocell systems," IEEE Communications Magazine, vol. 51, no. 5, pp. 178-185, May 2013.

[14] "Connecting the dots: small cells shape up for high-performance indoor radio," Ericsson Review, Dec 2014.

[15] S. H. R. Naqvi et al., "On the transport capability of LAN cables in allanalog MIMO-RoC fronthaul," in 2017 IEEE Wireless Communications and Networking Conference, March 2017.

[16] Y. Huang et al., "LTE over copper - potential and limitations," in Personal, Indoor, and Mobile Radio Communications (PIMRC), Aug 2015, pp. 1339-1343. 
[17] — "Enabling DSL and radio on the same copper pair," in 2015 IEEE International Conference on, June, pp. 1031-1035.

[18] E. Medeiros et al., "Crosstalk mitigation for LTE-over-copper in downlink direction," IEEE Communications Letters, vol. 20, no. 7, pp. 14251428, July 2016.

[19] Y. Huang et al., "Time-domain precoding for LTE-over-copper systems," in 2016 IEEE International Conference on Communications (ICC), May 2016, pp. 1-6.

[20] N. Carapellese et al., "Energy-efficient baseband unit placement in a fixed/mobile converged WDM aggregation network," IEEE Journal on Selected Areas in Communications, vol. 32, no. 8, pp. 1542-1551, Aug 2014.

[21] F. Musumeci et al., "On the placement of BBU hotels in an optical access/aggregation network for 5G transport," in Asia Communications and Photonics Conference 2015. Optical Society of America, 2015, p. ASu1G.2.

[22] T. Pfeiffer, "Next generation mobile fronthaul and midhaul architectures [invited]," IEEE/OSA Journal of Optical Communications and Networking, vol. 7, no. 11, pp. B38-B45, November 2015.

[23] J. Bartelt et al., "Fronthaul and backhaul requirements of flexibly centralized radio access networks," IEEE Wireless Communications, vol. 22, no. 5, pp. 105-111, October 2015 .

[24] D. Chitimalla et al., "5G fronthaul-latency and jitter studies of CPRI over Ethernet," IEEE/OSA Journal of Optical Communications and Networking, vol. 9, no. 2, pp. 172-182, Feb 2017.

[25] R. Acedo-Hernandez et al., "Automatic clustering algorithms for indoor site selection in LTE," EURASIP Journal on Wireless Communications and Networking, vol. 2016, no. 1, p. 87, 2016. [Online]. Available: http://dx.doi.org/10.1186/s13638-016-0587-3

[26] F. Tonini et al., "Optimization of centralized radio access networks in indoor areas," Proc. IEEE 18th International Conference on Transparent Optical Networks (ICTON) 2016.

[27] "Cpri specification v6.0," (2013-08-30).

[28] "C-RAN - the road towards green RAN," China Mobile White Paper, Version 3.0 (Dec 2013)

[29] K. M. Yang, "Encyclopedia of algorithms," Springer, 2008.

[30] V. V. Vazirani, "Approximation algorithms," Springer Science \& Business Media, 2013.

[31] T. H. Cormen et al., "Introduction to algorithms," MIT Press and McGraw-Hill, 2001.

[32] "http://www.ericsson.com/res/investors/docs/2013/ericsson-radio-dotsystem-telebriefings-26-sept.pdf."

[33] IBM ILOG CPLEX Optimization Studio V12.6.3.

[34] M. Mahloo et al., "Cost modeling of backhaul for mobile networks," in Communications Workshops (ICC), 2014 IEEE International Conference on, June, pp. 397-402.

[35] A. A. Widaa et al., "Toward capacity-efficient, cost-efficient and powerefficient deployment strategy for indoor mobile broadband," International Telecommunications Society (ITS), Tech. Rep., 2013.

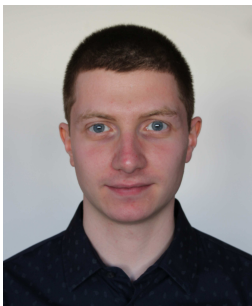

Federico Tonini received his B.S. degree in Electronics and Telecommunications Engineering and the M.S. degree in Telecommunication Engineering from University of Bologna in 2012 and 2015, respectively. He is currently pursuing his Ph.D. in Electronics Engineering, Telecommunications, and Information Technology at DEI, University of Bologna. His research interests include $5 \mathrm{G}$ communication system, network design and optimization techniques.

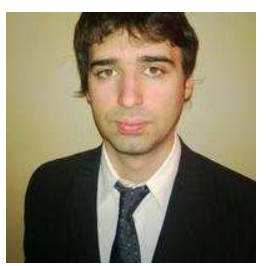

Matteo Fiorani received a PhD in ICT from University of Modena, Italy. During his PhD studies, he spent one year at Vienna University of Technology, Austria. From January 2014 to October 2016, he was a Postdoctoral Researcher a KTH Royal Institute of Technology, Stockholm, Sweden. He is currently Senior Researcher at Ericsson Research, Stockholm, Sweden. He was a Visiting Researcher at University of California, Davis and Columbia University, New York, USA. He is currently involved in $5 \mathrm{G}$ standardization in 3GPP and in several European and Swedish research projects. He has coauthored more than 50 peer-reviewed research papers (22 journal papers). His research interests include $5 \mathrm{G}$ radio and transport technologies.

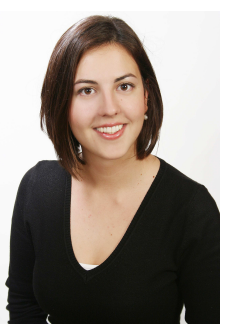

Marija Furdek received her Dipl.-Ing. (2008) and $\mathrm{Ph} . \mathrm{D}$. degrees (2012) in telecommunications from the University of Zagreb. Since 2013, she has been with the Optical Networks Lab (ONLab) at KTH. She was a visiting researcher at Massachusetts Institute of Technology, USA, and Auckland University of Technology, New Zealand. Her research interests include transport network planning and optimization, network resiliency and security. She has co-authored more than 50 publications in international journals and conferences, three of which received best paper awards. She is serving as a General Co-Chair of the Photonic Networks and Devices Conference, a part of the OSA Advanced Photonics Congress. She was the Publication Chair of IEEE OnlineGreenCom 2016 and IEEE ONDM 2014, and a Guest Editor of a special issue of the IEEE/OSA Journal of Optical Communications and Networking.

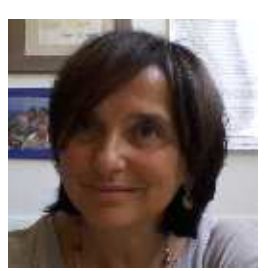

Carla Raffaelli is an Associate Professor at the University of Bologna. She received her M.Sc. and $\mathrm{Ph} . \mathrm{D}$ degrees in electronic and computer engineering (University of Bologna, Italy), in 1985 and 1990, respectively. She regularly acts as a reviewer for top international conferences and journals. She served as a member of Technical Program Committees in several Top International Conferences and served as Technical Program Committee co-Chair in ONDM 2011. She is a member of the editorial board of the journal Photonic Network Communications by Springer. She is IEEE Senior Member. She is the Director of the International Telecommunications Engineering Master's Degree at the University of Bologna, Italy. Her research interests include performance analysis of telecommunication networks, switch architectures, optical networks and 5G networks.

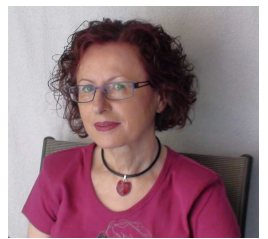

Lena Wosinska is a Full Professor at KTH Royal Institute of Technology. She is founder and leader of the Optical Networks Lab (ONLab). She has been working in several EU projects and coordinating a number of national and international research projects. Her research interests include fiber access and $5 \mathrm{G}$ transport networks, energy efficient optical networks, photonics in switching, optical network control, reliability and survivability, and optical datacenter networks. She has been involved in many professional activities including guest editorship of IEEE, OSA, Elsevier and Springer journals, serving as General Chair and CoChair of several IEEE, OSA and SPIE conferences and workshops, serving in TPC of many conferences, as well as being reviewer for scientific journals and project proposals. She has been an Associate Editor of OSA Journal of Optical Networking and IEEE/OSA Journal of Optical Communications and Networking. Currently she is serving on the Editorial Board of Springer Photonic Networks Communication Journal and of Wiley Transactions on Emerging Telecommunications Technologies. She is IEEE Senior Member.

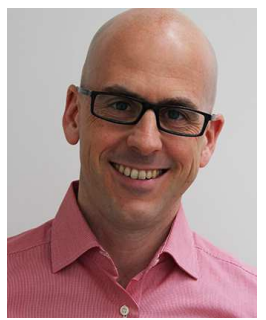

Paolo Monti is an Associate Professor at $\mathrm{KTH}$ Royal Institute of Technology. He is currently serving on the editorial boards of the IEEE Transactions on Green Communications and Networking and of the Springer Photonic Network Communications journal. He co-authored more than 120 technical papers, with three best paper awards. Dr. Monti regularly participates in several TPCs including IEEE Globecom and IEEE ICC. Among others, he also served as TPC co-chair of ONDM 2014, of IEEE OnlineGreenComm 2016, of HPSR 2017, of the ONS symposium at IEEE Globecom 2017, and of the OGN symposium at ICNC 2014, 2016 and 2017. His main research interests are within the networking and sustainability aspects of all-optical networks, with a special focus on optical transport solutions for $5 \mathrm{G}$ networks. Dr. Monti is a Senior Member of the IEEE. 